\title{
(An)Ästhetiken des Konsums. Inszenierungen von Warenwelt und (Super-)Marktgesellschaft bei Diamela Eltit und Annie Ernaux
}

\section{1 Ökonomisches erzählen: (An)Ästhetische und narrative Dimensionen von Märkten und Waren in der Moderne}

Von der Formierung der ersten frühkapitalistischen Gesellschaften bis in die unmittelbare Gegenwart hinein haben die beiden Gegenstandsbereiche der Ökonomie und der Literatur ein gemeinsames diskurshistorisches Feld komplexer Überlagerungen und wechselseitiger Bezugnahmen konstituiert. ${ }^{1}$ Insbesondere die Frage nach der ambivalenten Rolle ästhetischer Dispositive hat im Zusammenhang mit der sich seit dem 19. Jahrhundert herausbildenden Marktgesellschaft und einer sich intensivierenden Warenproduktion und Konsumaktivität eine besondere Aufmerksamkeit von Seiten der Kultur- und Wirtschaftstheorie wie auch der verschiedenen Künste und der Literatur im Besonderen erfahren. Die folgenden Betrachtungen wollen diesen - hier zunächst theoretisch wie historisch ausführlich zu klärenden Aspekt des Ästhetischen (bzw. des Anästhetischen) der Waren- und Konsumwelt in den Marktgesellschaften der spätkapitalistischen Gegenwart des 21. Jahrhunderts anhand zweier literarischer Texte untersuchen, die sich der Exploration des Supermarkts als einem paradigmatischen Ort verdichteter Beobachtung der Funktionsprinzipien dieser Marktgesellschaft verschreiben. Es handelt sich dabei um den Roman Mano de obra (2002) der chilenischen Autorin Diamela Eltit und den 2014 publizierten Text Regarde les lumières, mon amour der französischen Schriftstellerin Annie Ernaux. Vor der Analyse der beiden Werke gilt es jedoch zunächst, die (an)ästhetischen und narrativen Dimensionen von Märkten und Waren in der Moderne sowie den Zusammenhang zwischen Ästhetik und Konsum, exempla-

1 Als Beleg sowohl der historischen wie theoretischen Tiefe dieses Verhältnisses als auch der weiterhin ungebrochenen Produktivität seiner kulturwissenschaftlichen Erschließungsprozesse vgl. etwa die zahlreichen Beiträge des unlängst erschienenen Handbuchs Literatur \& Ökonomie (Vogl/Wolf 2020).

Ә Open Access. (C 2021 Benjamin Loy, publiziert von De Gruyter. (c))BY-NC-ND Dieses Werk ist lizenziert unter einer Creative Commons Namensnennung - Nicht-kommerziell - Keine Bearbeitung 4.0 International Lizenz. 
risch illustriert an der Institution des Supermarkts, theoretisch und historisch zu beleuchten.

Die Entstehung kapitalistisch geprägter Marktwirtschaften hat die Gesellschaften und ihre Strukturen wie kaum ein anderes Phänomen auf radikale Weise beeinflusst und verändert. Insbesondere ab dem 19. Jahrhundert intensiviert sich dieser von Karl Polanyi als Great Transformation beschriebene Prozess dahingehend, dass die vormals als ein gesellschaftlicher Teilbereich unter vielen fungierende Sphäre des Ökonomischen zum Ordnungsprinzip des Sozialen schlechthin wird und gewissermaßen ihren eigenen Gesellschaftstypus in Gestalt der Marktgesellschaft hervorbringt, denn:

[T] he control of the economic system by the market is of overwhelming consequence to the whole organization of society: it means no less than the running of society as an adjunct to the market. Instead of economy being embedded in social relations, social relations are embedded in the economic system. The vital importance of the economic factor to the existence of society precludes any other result. For once the economic system is organized in separate institutions, based on specific motives and conferring a special status, society must be shaped in such a manner as to allow that system to function according to its own laws. This is the meaning of the familiar assertion that a market economy can function only in a market society.

(Polanyi 2001 [1944], 60)

Die ungebrochene Relevanz dieses Verhältnisses zwischen Markt und Gesellschaft liegt aus kultur- und erzähltheoretischer Perspektive nicht zuletzt in der Tatsache begründet, dass der historische Aufstieg des Marktes und seine gegenwärtigen Rechtfertigungen von einem spezifischen Narrativ bzw. einem Welt- und Menschenbild begleitet werden, welches Joseph Vogl unter dem Begriff der ,Oikodizee erfasst hat. Diese bezeichnet einen in der Doktrin der klassischen Ökonomen geprägten und bis heute dominanten Ideenkomplex, demzufolge „Ausgleich und Gleichgewicht als wichtigster Beitrag ökonomischen Wissens zum allgemeinen Verständnis von sozialen Prozessen begriffen [werden] und [...] mit der liberalen Idylle des Marktes zugleich eine Art evolutionärer Fabel der Marktgesellschaft [liefern]“ (Vogl 2010, 53). Das Erzählen erhält folglich in der Sphäre der Ökonomie auf allen Ebenen einen zentralen Stellenwert, kommt ihm doch - über die Wirtschaftstheorie hinaus - in kapitalistischen Marktwirtschaften auf vielfältige Weise eine gleichermaßen dynamisierende und stabilisierende Funktion zu. So hängt etwa die grundlegende „dynamische Stabilisierung“ des Kapitalismus (vgl. dazu Rosa 2019, 671) fundamental von seiner spezifischen zeitlichen Orientierung auf eine imaginierte Zukunft hin ab, welche wiederum, wie Jens Beckert dargelegt hat, auf der Ebene der wirtschaftlichen Akteure elementar bestimmt wird von sog. „fictional expectations“. Im Gegensatz zur lange vorherrschenden soziologischen Tendenz, die Durchsetzung des Kapitalismus vor allem als Ergebnis seiner umfassenden Rationalisierungserfolge 
$\mathrm{zu}$ interpretieren, ${ }^{2}$ führt Beckert den überzeugenden Nachweis, dass die Dynamik kapitalistischer Ökonomien grundlegend von den Erwartungshaltungen ihrer Akteure abhängt. Diese wiederum seien im Kern eben nicht (rein) rational strukturiert, sondern fußten vor allem auf imaginären, affektiven und narrativen Faktoren und Prägungen, weshalb die Funktionsweise des Kapitalismus wesentlich als ein „secular enchantment of the world“ zu erfassen sei (Beckert 2016, 283). Dem Erzählen eignet in der Sphäre des Ökonomischen folglich eine fundamentale Ambivalenz: Einerseits ermöglichen narrative Strategien die stetige Dynamisierung des Systems im Sinne einer fortwährenden Schaffung prospektiv orientierter Bedürfnis- und Produktionsnarrative; andererseits ermöglichen die gleichzeitig zur Beherrschung der systemischen Instabilitäten entworfenen Anpassungserzählungen die Schaffung von Stabilitäts- und Harmonienarrativen. Vor allem letztere beziehen in Marktgesellschaften - sowohl auf systemischer als auch auf individueller (Handlungs-)Ebene - einen beträchtlichen Teil ihrer vermeintlich rationalen Begründungslogiken aus vor- bzw. irrationalen Reservoiren. ${ }^{3}$ Erzählungen des Ökonomischen sind also - von den klassischen Modellen des 18. Jahrhunderts bis zu den neoliberalen Konzepten des 20. und 21. Jahrhunderts ${ }^{4}-$ immer $^{2}$ schon einer grundlegenden Paradoxie, um nicht zu sagen einer bewussten Rhetorik der Verschleierung unterworfen. Die metaphysisch und teleologisch grundierten Autorisierungsnarrative des Kapitalismus, also etwa die Proklamierung seiner vermeintlichen Stabilität oder die Macht seiner allgemeinen Wohlstandsallokation, machen sich die basalen Vorteile jedes Erzählens im Sinne von Kontingenzreduktion und Entdifferenzierung ${ }^{5}$ zunutze, um seine eigene Instabilität ${ }^{6}$, seine imminent destruktiven Dimensionen sowie seine

2 Vgl. hierzu paradigmatisch etwa die Thesen Max Webers, der sich darum bemühte, die westliche „rational-kapitalistische Organisation“ gegenüber allen dieser vermeintlich entgegengesetzten und an „gewaltpolitischen oder an irrationalen Spekulationschancen“ (Weber 1989, 244-245) orientierten Phänomenen abzugrenzen.

3 So bemerkt etwa Joseph Vogl schon mit Blick auf die Entstehung der frühmodernen ,Theorien“ der klassischen Ökonomie, diese seien vom „Herniedersinken theologischer und kosmologischer Fragen in das Gebiet der Sozialontologie“ geprägt gewesen (Vogl 2010, 41).

4 Vgl. dazu auch Vogl 2010, 83-114, sowie die rezenten und erhellenden Studien zu den ideengeschichtlichen bzw. anwendungspraktischen Ursprüngen des Neoliberalismus von Slobodian 2018 und Chamayou 2018.

5 Vgl. hierzu etwa die grundlegenden Ausführungen von Koschorke 2012, 27-110.

6 Vgl. hierzu den Kommentar von Beckert: „Only when the fictional character of expectations and of transcending qualities is hidden do actors feel comfortable enough to make decisions whose outcomes are by nature unpredictable or based on the attribution of qualities that exist only as contingent meanings“ (Beckert 2016, 73). 
problematischen systemischen Prämissen, Motivationen und Funktionsweisen zu marginalisieren oder gänzlich auszublenden. ${ }^{7}$

Diese Bedeutung der erzählerischen Steuerung wird besonders augenfällig im zentralen Element und Motor allen marktwirtschaftlichen Geschehens: der Produktion von Waren und ihrer spezifischen Ästhetik. Der Warenkonsum bildet im Kontext einer umfassenden Ökonomisierung des Sozialen in der Moderne bekanntlich einerseits ein „konstitutives Moment moderner Vergesellschaftung“, da seine Funktion darin besteht, „Objekte zur Anzeige des sozialen Prestiges in einer sozial zunehmend mobilen, jedoch nicht egalitären Gesellschaft zu verwenden“ (Schrage 2009, 251). Andererseits intensivieren sich mit der radikalen Ausweitung der Verfügbarkeit von Waren als käuflich zu erwerbenden Dingen und Dienstleistungen im 19. Jahrhundert ${ }^{8}$ auch die an sie gebundenen ,imaginaries of consumption“ (Beckert 2016, 280), wird die Waren- und Konsumkultur ein zentraler Bestandteil der (parallel zum Prozess der Rationalisierung verlaufenden) allgemeinen Ästhetisierung des Sozialen in der Moderne. ${ }^{9}$ Die traditionelle marxistische Warenkritik $^{10}$ hat - ausgehend von Marx' Beobachtung im Kapital zum „Doppelcharakter der Ware“ als ein „Zwieschlächtiges, Gebrauchswert und Tauschwert“ (Marx 1962 [1867], 56) - diesen Aspekt hinlänglich adressiert, indem sie gezeigt hat, dass jedem Gebrauchswert einer Ware bereits ein ,Gebrauchswertversprechen` inhärent ist. Indem bei der Produktion einer Ware über ihren (Gebrauchs-)Wert ,als solchen' hinaus, wie Haug in seiner klassischen Kritik der Warenästhetik anführt, immer auch eine „Erscheinung des Gebrauchswertes“ (Haug 2017 [1971], 29) produ-

7 Gerade der letzte Aspekt wird in den jüngsten soziologischen und wirtschaftswissenschaftlichen Diskussionen verstärkt diskutiert, etwa wenn es um die Frage der Ausblendung der globalen Folgen und Asymmetrien kapitalistischer Liefer- und Warenketten bzw. des westlichen Lebens- und Konsumverhaltens im Sinne sog. „Externalisierungsgesellschaften“ geht (Lessenich 2016) oder der Aspekt der gleichfalls zentral ,erzählungsbasierten“ Wertbildung als solcher in kapitalistischen Gesellschaften problematisiert wird (Mazzucato 2019).

8 Die Anfänge dieser nicht zuletzt mit einer beschleunigten Globalisierung einhergehenden Verfügbarkeit von Dingen lassen sich bereits im 18. Jahrhundert intensiv beobachten (vgl. etwa die Beiträge in Neumann 2015 oder, mit stärkerem Fokus auf den affektiven Dimensionen, in Schlünder und Stahl 2018).

9 Auch hier hat die jüngere kultursoziologische Forschung entscheidende Anstöße geliefert, um die lange dominante Ansicht von der „Moderne als ein Sachzusammenhang formaler Rationalität“ zu hinterfragen bzw. auf der Grundlage eines erweiterten Konzepts des Ästhetischen sogar umzukehren, indem man die Tatsache in den Mittelpunkt gerückt hat, dass „[d]ie Lebensstile, die Ökonomie, ihre Formen der Arbeit und des Konsums, die modernen Medientechnologien, der Städtebau, die persönlichen Beziehungen, die Kultur des Selbst und des Körpers sowie teilweise auch das Politische und die Wissenschaft [...] zum Gegenstand von Prozessen der Ästhetisierung [werden]“ (Reckwitz 2017a, 13-14).

10 Vgl. dazu etwa die theoriegeschichtlichen Überlegungen von Eiden-Offe 2020. 
ziert wird, ${ }^{11}$ die dem in Abhängigkeit von der Käuferperspektive individuell variablen und somit bis zu einem gewissen Grade von der Ware selbst unabhängigen Wert entspricht, liegt in der Warenproduktion ein

ökonomisch funktioneller Akzent auf der Erscheinung des Gebrauchswerts, der, den einzelnen Kaufakt betrachtet, tendenziell als bloßer Schein eine Rolle spielt. Das Ästhetische der Ware im weitesten Sinne: sinnliche Erscheinung und Sinn ihres Gebrauchswerts, löst sich hier von der Sache ab. Schein wird für den Vollzug des Kaufakts so wichtig - und faktisch wichtiger - als Sein.

(Haug 2017 [1971], 29)

Dies bedeutet, dass das Sinnliche „zum Träger einer ökonomischen Funktion“ (Haug 2017 [1971], 30) wird und damit die ästhetische Affizierung des Käufers durch die sinnlich wahrgenommene Erscheinung der Ware zunehmend in den Mittelpunkt rückt. Bereits Walter Benjamin notiert diesen Punkt im Passagen-Werk, wenn er in den „dramatischen Aufschriften der magasins de nouveautés [...] die Kunst in den Dienst des Kaufmanns“ (Benjamin 1983, 86) treten sieht und „das circensische und schaustückhafte Element des Handels“ (93) hervorhebt. Die Kritik der Warenästhetik richtet sich nun - ähnlich wie die Hinterfragung der Narrative der ,Oikodizee، - gegen die (aus ihrer Sicht) manipulative Macht jener ,Versinnlichung' der Ware, ${ }^{12}$ die sich gleichermaßen durch ihre künstl(er)i(s)che Darbietung und die verschleiernde Kraft der Sprache bzw. ihrer erzählerischen Mittel entfaltet. ${ }^{13}$ Diese Ästhetisierung der Ware - Haug spricht von der „Hinterhältigkeit in der Schmeichelei der Waren“ (84) - erfüllt aus Sicht der traditionellen marxisti-

11 Haug führt als Beispiel etwa den Bereich der Nahrung an, wo die Annahme der Existenz eines ,reinen“ Gebrauchswerts etwa „nur solange plausibel [erscheint], als bei ,Gebrauchswert“ etwa von Lebensmitteln an so etwas wie elementare Nährstoffe o. Ä. gedacht wird. Doch sobald Nahrung kulturell artikuliert ist, besitzt sie die im Wortsinn mythische Dimension, dass sie einem etwas ,bedeutet‘ und gleichsam in eine ,Erzählung، eingehüllt ist“ (Haug 2017, 332).

12 Vgl. dazu auch die wichtige Bemerkung von Haug, die Kritik richte sich „nicht gegen die Verschönerung bestimmter Dinge, ergeht sich schon gar nicht in der Genussfeindschaft, verklärt auch nicht den Staatssozialismus, den sie als Kontrastfolie heranzieht, sondern sie zeigt, wie eine losgelassene ökonomische Funktion des Kapitalismus mit der Macht einer Naturkatastrophe durch die sinnliche Welt fegt und alles, was sich ihr nicht fügt, niederwirft, dagegen das, was ihr entgegenkommt, aufnimmt, ungeheuer verstärkt und zur Herrschaft erhebt, um die des Kapitals zu befestigen“ (Haug 2017 [1971], 180).

13 Auch dieser Aspekt findet sich - auf einer gleichwohl eher systemischen Ebene - bereits in Benjamins Fragmenten, wenn er über die zensurbasierte sprachliche Aufrechterhaltung einer nur scheinhaften ökonomischen Stabilität in der Börsenberichterstattung im Paris des frühen 20. Jahrhunderts notiert: „Ruf der Verkäufer der Kurszettel auf der Straße: bei Hausse ,La hausse de la Bourse‘. Bei Baisse: ,Les variations de la Bourse‘. Der Terminus ,Baisse‘ war polizeilich verboten“ (Benjamin 1983, 88). Dass sich dieser Konnex zwischen Ökonomie und Erzählung seitdem nicht etwa abgeschwächt, sondern vielmehr in Gestalt eines heute in allen großen Firmen einen zentralen Platz einnehmenden storytelling noch intensiviert hat, lässt 
schen Warenästhetik folglich die Funktion, die ewige Steigerungsdynamik des Kapitalismus unter Wahrung der asymmetrischen Besitzverhältnisse durch den gewissermaßen ,besinnungslosen' Konsum aller aufrecht zu erhalten, welcher eben durch die mangelnde Einsicht der Konsumenten in diese ästhetischen Funktionsmechanismen als Teil des ,Verblendungszusammenhangs' des kapitalistischen Systems erst ermöglicht bzw. beständig befördert wird.

Das Ästhetische, dem im Kontext der Warengesellschaft die grundlegende Aufgabe einer „Zurichtung der Sinnlichkeit“ (Haug 2017 [1971], 185) zufällt, lässt sich jedoch aus dieser Perspektive zugleich als ein Phänomen bzw. eine Strategie bestimmen, die sich an der Grenze zum Anästhetischen bewegt, also gewissermaßen einer intendierten ,Empfindungslosigkeit‘ zuarbeitet (im Sinne einer mangelnden Hinterfragung von Bedingungen und Zwecken bestimmter Sinneswahrnehmungen im ökonomisch-sozialen Kontext). Dieser Aspekt ist auch in der gegenwärtigen ästhetischen Philosophie von unterschiedlichen Arbeiten problematisiert worden. So hat etwa Wolfgang Welsch in diesem Zusammenhang in seinen wahrnehmungstheoretischen Überlegungen darauf hingewiesen, dass prinzipiell jedem „Wahrnehmen selbst eine Art Anästhetik eingeschrieben ist. Seine eigene Spezifizität - seine Schemata und Prägungen einschließlich der damit gesetzten Beschränkungen - bleibt ihm eigentümlich verborgen. Und diese interne Anästhetik ist eine notwendige Bedingung der externen Effizienz des Wahrnehmens“ (Welsch 2017, 38). Über diese gleichsam anthropologische und physiologische Dimension hinaus jedoch postuliert Welsch die konkrete Wirksamkeit dieser Dialektik in allen ,Bildbereichen“ von Gesellschaftlichkeit, in denen Prinzipien des Ästhetischen operieren bzw. wo Bilder bewusst sich „die Tarnkappe des Anästhetischen übergezogen, in anästhetische Latenz sich begeben haben“ und damit „,verbindlich“, d.h. zwingend“ geworden seien (Welsch 2017, 39). Die Aufgabe der Kunst sei es nun - und hier ließe sich an ähnlich gelagerte Konzepte wie Jacques Rancières Idee von der Literatur als Eingriff in die „Einteilung der Räume und der Zeiten, des Sichtbaren und des Unsichtbaren, der Sprache und des Lärms“ (2011, 14) denken -, „solche Latenzen aufzubrechen“, um mittels einer „Konfrontation mit derlei abweichenden Perzeptions- und Gestaltungsformen [zu lehren], wie sehr ästhetische Erwartungshaltungen eingeschliffen sind und in ihrer Selbstverständlichkeit durch eine eigentümliche Anästhetik gedeckt werden“ (Welsch 2017, 40-41). Welschs Idee einer (An)Ästhetik geht also ebenso wie Rancières Konzept des partage du sensible von der Annahme aus, dass Wahrnehmung grundsätzlich durch ein „historisch und

sich etwa mit Philipp Schönthalers erhellendem Essay Portrait des Managers als junger Autor (2016) anschaulich nachvollziehen. 
kulturell spezifisches Apriori vorstrukturiert“ bzw. „genauso wie das begriffliche Denken kulturell begrenzt [ist]“ und somit „bestimmte Teile der sozialen Wirklichkeit systematisch aus[klammert]“ (Prinz 2017, 365).

In der traditionellen marxistischen Kritik von Lukács über Adorno bis zu Haug koinzidieren die Diagnosen des (An)Ästhetischen der Ware letztlich immer in der - angesichts ihrer gleichzeitig postulierten Sinnesmacht etwas paradoxen - Feststellung, der Ware mangele es „sowohl in sinnlicher als auch in kognitiver Hinsicht an Subtilität und Komplexität“ (Drügh 2015, 125). Das „Zur-Ware-werden aller Gegenstände“ mündet aus dieser Perspektive notwendig in eine als Entfremdung empfundene Verdinglichung des seiner „organischen Einheit der Person“ beraubten Menschen (Lukács 1983 [1923], 194). Demgegenüber haben neuere kulturwissenschaftliche Ansätze, durchaus unter Rückgriff auf alternative Traditionslinien der Soziologie der Moderne, wie etwa Georg Simmels Philosophie des Geldes, versucht, gerade die produktiven ästhetischen Potenziale der Konsum- und Warensphäre stärker mit Blick auf ihre „Sensibilität und Wahrnehmungsfähigkeit für das Gegenwärtige“ bzw. als „kulturformende Kraft“ zu deuten (Drügh 2015, 125 bzw. 128). Waren werden aus dieser Perspektive vor allem auf ihre „kulturbildende, -ordnende und -interpretierende Funktion“ bzw. als „Orte gemeinschaftlicher Imagination“ untersucht (Drügh 2015, 14-15). ${ }^{14}$ Über die Analyse der allgemeinen Bedeutungen und Funktionsweisen ästhetischer Verfahren im Rahmen der Warenproduktion und des Konsums hinaus haben diese Studien vor allem für die Spätmoderne mit ihrer eigenen Erscheinungsform eines „ästhetischen Kapitalismus“ auch die spezifischen Orte und Akteure in den Blick genommen, von denen im folgenden Abschnitt nun die ,Institution“ des Supermarkts (und seine kulturelle Produktivität) noch einmal gesondert im Hinblick auf seine Bedeutung als Schauplatz der anschließend zu analysierenden Texte von Diamela Eltit und Annie Ernaux betrachtet werden soll.

\section{Logiken und (Nicht-)Orte des ästhetischen Kapitalismus: (Super-)Markt-Fiktionen der Spätmoderne}

Was für die sog. „organisierte, industrielle Moderne, die von etwa 1920 bis Mitte/ Ende der 1970er Jahre reicht“ (Reckwitz 2017b, 100), in Hinblick auf die Rolle der

14 Vgl. zu diesem Aspekt auch die Überlegungen von Urban 2018, 135-144. 
Ästhetisierung des Sozialen und Ökonomischen noch in begrenztem Umfang galt, hat sich in den vergangenen fünfzig Jahren zu einem neuen Gesellschaftsund Wirtschaftsmodell verdichtet, das wahlweise mit Begriffen wie „Kulturkapitalismus“ (Reckwitz 2017b) oder „ästhetischer Kapitalismus“ (Böhme 2018) erfasst wurde. Die ästhetische und narrative Dimension der Warenproduktion der Moderne hat eine weitere Intensivierung erfahren, die in der globalisierten und digitalisierten Spätmoderne einen „Umschlag der Ökonomie markiert, nachdem das Ästhetische nicht mehr nur ein Gegenstand industrieller Produktion ist, sondern die Ökonomie selbst ergreift“ (Böhme 2018, 99). In den spätkapitalistischen Ökonomien mit ihrer umfassenden Deckung aller Grundbedürfnisse lässt sich Wachstum nurmehr „durch die Herstellung ästhetischer Werte“ erzeugen bzw. erfolgt ein „Wandel von Bedürfnissen in Begehrnisse“ (Böhme 2018, 11 bzw. 16), welche wiederum noch intensiver als je zuvor einer ästhetisch-erzählerischen Aufladung bedürfen, denn: „Kulturelle Güter haben häufig eine narrative und hermeneutische Qualität, indem sie die Form von Erzählungen annehmen, die für den Rezipienten bedeutungsvoll sind. Dass kulturelle Güter nicht selten Geschichten erzählen, kann gar nicht genug betont werden“ (Reckwitz 2017b, 121). Mit dieser Radikalisierung der ästhetischen Zeichen-Welt im Kontext einer neuen „Bereicherungsökonomie“ (Boltanski/Esquerre 2019) jedoch intensiviert sich zugleich, wie Jean Baudrillard bereits in seiner frühen Studie zur spätmodernen Konsumgesellschaft gezeigt hat, die Macht und Präsenz des Anästhetischen, da die konkreten Bedingungen und Folgen der Produktion und des Konsums von Waren vollends in einer Sphäre des „Magischen“ (im Sinne einer gleichsam behexenden oder eben anästhesierenden Wirkung) aufgelöst werden:

[C]'est une pensée magique qui régit la consommation, c'est une mentalité miraculeuse qui régit la vie quotidienne. [...] Dans la pratique quotidienne, les bienfaits de la consommation ne sont pas vécus comme résultant d'un travail ou d'un processus de production, ils sont vécus comme miracle. [...] Les biens de consommation se proposent ainsi comme de la puissance captée, non comme des produits travaillés. [...] Car, même si l'abondance se fait quotidienne et banale, elle reste vécue comme miracle quotidien, dans la mesure où elle apparaît non comme produite et arrachée, conquise, au terme d'un effort historique et social, mais comme dispensée par une instance mythologique bénéfique dont nous sommes les héritiers légitimes: la Technique, le Progrès, la Croissance, etc.

(Baudrillard 2017 [1970], 27-29)

Als einen paradigmatischen Ort dieser spätmodernen und (an)ästhetisierten Marktgesellschaft identifiziert schon Baudrillard den Supermarkt bzw. das Einkaufszentrum, wo nicht nur eine Amalgamierung der (Waren-)Zeichen erfolgt, sondern eine neue total(itär)e Erfahrung der Verschmelzung von Konsum und Leben möglich wird „dans une évolution qui va de l'abondance pure et simple, à travers les réseaux articulés d'objets jusqu'au conditionnement 
total des actes et du temps, jusqu'au réseau d'ambiance systématique inscrit dans les cités futures que sont les drugstores, les Parly 2 ou les aéroport modernes“ (23-24). Der Super- oder Hypermarkt bzw. das Einkaufszentrum werden damit zum Lern- und Praxisort einer spezifischen Subjektivierungsarbeit, die der Neoliberalismus im Rahmen seiner ihm eigenen Gouvernementalität dem konsumierenden Subjekt nicht nur durch politische oder rechtliche Interventionen verordnet, ${ }^{15}$ sondern auch durch (an)ästhetische (Konsum-)Erfahrungen gleichsam unterbewusst antrainiert. Als ein solcher Ort wird der Supermarkt zu einem privilegierten Schau-Platz der Verhandlung von Ökonomie und Gesellschaftlichkeit sowie der (an)ästhetischen Phänomene, die in diesen Sphären wirksam sind. Dabei ist diese Form des Einkaufens, die sich in den USA ab den 1930er, in Europa ab den späten 1950er und in Lateinamerika ab den 1970er Jahren durchsetzt, einmal mehr von einer für das (An)Ästhetische konstitutiven Ambivalenz geprägt: einerseits durch eine effizienz- und kontrollbasierte sowie auf Tempomaximierung ausgerichtete Funktionsweise, die ihre räumliche und sinnliche Entsprechung in der „Funktionsästhetik“ (Drügh 2015, 109) der Discounter findet; und andererseits durch eine diese Zweckrationalität zunehmend überschreitende Ästhetisierung der Konsumsphäre, wie sie exemplarisch in Malls und Einkaufszentren realisiert wird, welche an die ursprünglich dem klassischen Warenhaus der Moderne bzw. den Passagen vorbehaltenen ästhetischen Prinzipien anknüpfen (Böhme 2018, 117).

Der Beobachtung der (markt)gesellschaftlichen Relevanz dieser Einkaufsorte steht allerdings die Tatsache einer „extraordinary infrequency“ von Supermarkt-Fiktionen gegenüber, ,given their prominence in daily life and in cultural metaphor for nearly half the century“ (Bowlby 2001, 195). Wenngleich ein gewisser ,Kanon` an Supermarktfiktionen in den einschlägigen Studien zum Thema beobachtbar ist, der von Zolas Warenhaus-Klassiker Au bonheur des dames über US-amerikanische Beispiele wie Don DeLillos White Noise oder Allen Ginsbergs A Supermarket in California bis hin zu Andy Warhol oder den Romanen Michel Houellebecqs reicht, scheint die allgemein geringe Präsenz solcher Konsumorte in den Fiktionen der (Spät-)Moderne eng mit einem dominanten modernistischen Selbstverständnis von Kunst zusammenzuhängen, d. h. im Sinne eines „diskursiven Raum[es], von dem aus man den leidenschaftlichen Interessen des Marktes das interesselose Wohlgefallen der Kunst und also den Warenwerten die wahren Werte entgegenzusetzen behauptet[e]“ (Drügh 2015, 128). Auffällig ist in jedem Fall, dass dort, wo Fiktionen sich der Waren- und Konsumsphäre widmen,

15 Vgl. dazu grundlegend Michel Foucaults Überlegungen zur Geschichte der Gouvernementalität bzw. auch die erhellende Studie von Marchart 2013 zur Prekarisierung als bedeutendem Effekt dieser neoliberalen (Selbst-)Techniken. 
Orte wie die Einkaufspassage, der Supermarkt oder die Shopping-Mall immer schon als metonymischer Verhandlungsraum von Sozialität und zugleich als eine autonome Eigenwelt des Sozialen - die Passage sei „eine Stadt, eine Welt im Kleinen“, notiert bereits Benjamin $(1983,83)^{16}$ - mit eigenen Gesetzmäßigkeiten und (an)ästhetischen Dimensionen dargestellt werden: Im Warenhaus wie im Einkaufszentrum verdichten sich die „Parameter der Konsumgesellschaft [...] sinnfällig in einem homogenen und transparenten Raum“ (Urban 2018, 135), wobei zu betonen ist, dass die für die Warenwelt eingangs bestimmte Ambivalenz auch für die Orte des Konsums gilt. Der Supermarkt - und die im Folgenden zu untersuchenden Texte belegen dies - vermag gleichermaßen als ,paragesellschaftliche‘ Sphäre von Gewalt, Entfremdung und sozialer Segregationserfahrung $\mathrm{zu}$ fungieren wie auch als gesellschaftlicher Kreuzungs- und Begegnungsort, der sich mithin sogar als „einer der letzten, wenn nicht der letzte effektive Sinngenerator in einer Gesellschaft [erweist], die die für ihren Zusammenhalt notwendige integrative Energie allein aus dem mit- und nebeneinander [...] vollzogenen Konsum bezieht“ (Urban 2018, 138).

Der Supermarkt und das Einkaufszentrum bilden folglich aufgrund ihrer Eigenschaft, „[to] give topographical form to the social, and [...] visualize abstract socialtheoretical dynamics, such as agency“ (Alwort 2010, 323), einen privilegierten Beobachtungs- und Inszenierungsort für literarische (und filmische) ${ }^{17}$ Verhandlungen spätmoderner Gesellschaftlichkeit und der sozialstrukturierenden Rolle von Konsum und Warenwelt. Zugleich stellt sich die Frage, wie speziell literarische Texte mit der Tatsache umgehen, dass zwischen den (An)Ästhetisierungsoperationen des spätmodernen Kapitalismus und den eigenen ästhetischen Darstellungsund Formverfahren nicht nur Berührungspunkte bestehen, sondern die (Sprach-) Kunst einen elementaren Beitrag zum Funktionieren dieser kulturellen Ökonomie leistet bzw. ja selbst gewissermaßen seit Beginn der Moderne das Modell für die „Singularitätsmärkte“ (Reckwitz 2017b, 155) der Gegenwart geliefert hat. ${ }^{18}$ Vor diesem Hintergrund sollen im Folgenden die beiden Texte Mano de obra von

16 Vgl. auch die Bemerkung von Bowlby, wonach Supermärkte und Warenhäuser ,as emblematic of contemporary developments not only in marketing, but in social life more generally“ zu betrachten seien $(2001,8)$.

17 Vgl. dazu etwa die Analysen zu Harun Farocki in Drügh 2015 bzw. weitergehend zu filmischen Inszenierungen neoliberaler Gesellschaftlichkeit in Lateinamerika die Studien von Loy 2017 bzw. Urban 2018.

18 Vgl. dazu ausführlich die Beobachtung von Reckwitz, dass gerade entgegen einer dominanten idealistischen Auffassung moderner Kunst- und Literaturphilosophie die Kunst mit der Moderne nicht für das Andere der Ökonomie stehe, sondern „seit dem Ende des 18. Jahrhunderts die Strukturmerkmale einer Ökonomie der Singularitäten entfaltet hat. [...] Die Kunst brauchte nicht erst nachträglich - etwa durch die Kulturindustrie seit den 1920er Jahren oder den globa- 
Diamela Eltit und Regarde les lumières, mon amour von Annie Ernaux im Hinblick auf ihre literarischen Verfahren zur Darstellung gegenwärtiger (Super-) Marktgesellschaften analysiert werden, welche jeweils spezifische Formen der Raum- und Zeitwahrnehmung, der Semantisierung und Symbolisierung sowie der Meta-Reflexivität über den Zusammenhang zwischen der (An)Ästhetik des Warenkonsums und den (un-)möglichen Formen spätmoderner Subjektivität und Sozialität umfassen.

\section{Paria der Marktgesellschaft: (An)Ästhetiken neoliberaler Anomie in Diamela Eltits Mano de obra (2002)}

In wohl keinem anderen Land der Welt wurde eine ganze Gesellschaft auf eine vergleichbar radikale Weise ökonomisch ,reformiert' wie im Falle Chiles unter der von 1973 bis 1990 von Augusto Pinochet angeführten Militärregierung, deren sozio-ökonomisches Projekt Naomi Klein einmal als den „most extreme capitalist make-over ever attempted anywhere“ bezeichnet hat (Klein 2007, 15). Unter autokratischen Rahmenbedingungen wurde die fundamentalistische neoliberale Doktorin der sog. ,Chicago Boys“, einer Gruppe an der University of Chicago unter Milton Friedman ausgebildeten chilenischen Ökonomen, im Zuge einer ,Schock-Strategie' und in Form von umfassenden Privatisierungen quasi aller Bereiche öffentlicher Daseinsvorsorge wie Bildung, Gesundheit oder des Rentensystems implementiert. Zugleich erfolgte ab den späten 1980er Jahren über eine extreme Ausweitung von Verbraucherkrediten und neuen Formen, Orten und Medien ${ }^{19}$ des Massenkonsums in Gestalt von Shopping-Malls und Hypermärkten die Etablierung einer ungekannten Konsumkultur, die - wie der chilenische Soziologe Tomás Moulian gezeigt hat - nicht nur von spezifischen modernisierungsbasierten Rechtfertigungsnarrativen begleitet wurde (Moulian 2002, 99), sondern

len Kunstmarkt seit den 1990ern - ökonomisiert werden, sie war von Anfang an in einer Drastik vermarktlicht, das heißt kulturökonomisiert, wie kein anderes Feld der modernen Gesellschaft“ (2017b, 155-156).

19 Vgl. zu dieser für ganz Lateinamerika bedeutsamen Dimension und den radikalen Transformationen der politisch-sozialen Systeme und Leitbegriffe vom ciudadano zum consumidor auch die einschlägigen Beobachtungen von Nestor García Canclini: „Esta reestructuración de las prácticas económicas y culturales conduce a una concentración hermética de las deciciones en élites tecnológico-económicas y genera un nuevo régimen de exclusión de las mayorías incorporadas como clientes“ $(1995,41)$. 
zugleich profunde transformatorische Effekte bezüglich sozialer Subjektivierungsprozesse des neuen „ciudadano credit-card“ hatte: „La cultura cotidiana del Chile Actual está penetrada por la simbólica del consumo. Desde el nivel de la subjetividad esto significa que en gran medida la identidad del Yo se constituye a través de los objetos“ (Moulian 2002, 103 bzw. 106). Die überall im Land entstehenden Shopping-Malls und Hypermärkte werden dabei zu einem „gran escenario de sublimación, de idealización del consumo“ (Moulian 2002, 114), also zum neuen bevorzugten Ort einer performativen Verhandlung von (Markt-)Gesellschaftlichkeit. Dieser übernimmt einmal mehr sowohl integrative als auch selektive Funktionen, indem er weiten Schichten der Gesellschaft neue, kreditbasierte Formen konsumistischer Partizipation eröffnet, während er eine gewisse Gruppe von „parias“ ausschließt, „cuyo comportamiento pasado y cuyo salario presente no lo[s] hacen acreedor[es] a la confianza financiera“ (Moulian 2002, 106).

Vor diesem Panorama spielt der 2002 publizierte Roman Mano de obra von Diamela Eltit, deren Werk wie kaum ein anderes seit den späten 1980er Jahren die Folgen dieser neoliberalen Revolution in Chile literarisch vermessen hat. Der Text schildert aus Sicht einer bzw. mehrerer nicht näher bestimmter Erzählinstanzen das Leben einer Gruppe von Supermarkt-Angestellten, wobei sich der erste Teil des Romans stärker auf die Darstellung der Tätigkeiten selbst konzentriert, während der zweite vor allem das konfliktgeprägte Zusammenleben der Gruppe von Männern und Frauen in einem baufälligen Haus fokussiert. Für die Frage der (An)Ästhetik des Warenkonsums ist hier zunächst relevant, dass der Super- bzw. Hypermarkt als Schauplatz sowie die Tätigkeiten der Angestellten umfassend von einem Zwang zur Ästhetisierung geprägt sind: So werden Kunden wie Angestellte als Figuren auf der bestens ausgeleuchteten ,Bühne‘ der Verkaufsflächen dargestellt (Eltit 2005 [2002], 32), wobei vor allem zweitere in ihrer „uniforme caricaturesco y su impúdica leyenda inscrita en mis espaldas y la obligación de la caminata incesante“ (Eltit 2005 [2002], 56) permanent zum Fingieren von Service-Bereitschaft und Aktivität angehalten sind. Neben dem geschilderten Ausbeutungssystem, das vor allem in der permanenten Überwachung durch Kameras und Aufseher sowie im basalen Funktionsprinzip der Disziplinierung durch jederzeit problemlos mögliche Kündigung besteht, sind die Körper der Angestellten immer auch Teil der vielfältigen Ästhetisierungsoperationen der Waren- und Marktwerbung, in deren Rahmen sie sich kostümieren oder als Tänzer auftreten müssen (,acostumbrados penosamente a que nos gritaran, que nos obligaran a disfrazarnos. Que nos vistieran de viejos pascueros en Navidad, de osos, de gorilas, de plantas, de loros, de pájaros locos los domingos. Que nos impusieran el deber de bailar cueca el 18, de bailar jota el 12 de Octubre“ (Eltit 2005 [2002], 111)). Ein Mitglied der Gruppe, Gabriel, wird gar als authentischer ,Warenkünstler beschrieben, dessen Talent in der ästhetisch ansprechenden Drapierung der Waren besteht 
- „cuando debía acumular, como si fueran esculturas, los altos de Cocas, las montañas de Pepsis [...] convertía a esa carne sanguinolenta en un espectáculo“ (Eltit 2005 [2002], 126-127); diese Kunst ist jedoch (im Wortsinne) ,brotlos', da ihm der Supermarkt kein Gehalt zahlt, sondern er als ,Verdienst' für seine ästhetische Arbeit lediglich das Recht erhält, an den Kassen als Wareneinpacker zu arbeiten. Die Szene verbildlicht gleichsam allegorisch den ambivalenten Status des Ästhetischen in der Warenwelt, fungieren Gabriels Präsentationsfähigkeiten doch gleichermaßen als Kundenmagnet und stets kontrollierte wie zweckgebundene Werke eines domestizierten Künstlers (,se encontraba atado al mesón como un animal de feria“, Eltit 2005 [2002], 128).

Hinter dieser Welt des ästhetischen Warenspektakels widmet sich der Roman vor allem der Darstellung der anästhesierenden Effekte der Arbeitsbedingungen in der neoliberalen Marktgesellschaft: Was die temporär verweilende Kundin als ansprechende Darbietung der Waren in Form bestimmter Licht- und Geräuschkulissen, als kunstvolle Anordnung von Produkten oder auch nur als banalen und knappen Bezahlkontakt wahrnimmt bzw. aufgrund des Gewohnheitseffekts eben nicht (mehr) bewusst vergegenwärtigt, wird aus der im Roman vorherrschenden Sicht der Angestellten des Supermarkts zu einer nicht endenden Erfahrung psychischer Entfremdung und körperlichen Verfalls. Hinter der ästhetisierten Oberfläche des Supermarkts verbirgt sich die von Émile Durkheim als „eine Art Dauerzustand [...] in der Welt des Handels und der Industrie“ (1983 [1897], 290) diagnostizierte Anomie, also jener „Zustand der gestörten Ordnung“ (289), den der französische Soziologe als unmittelbare Folge der radikalen Entfesselung der Ökonomie und der mit ihr verbundenen Affekte, Leidenschaften und Fantasien erfasste. Den aus der Anomie nach Durkheim resultierenden Suizid-Wunsch formuliert denn auch eine der Figuren explizit, wenn sie davon träumt, angesichts ihrer Erschöpfung unter den Waren begraben zu werden: „Mi deseo (mi último deseo) es derrumbarme en medio de un estrépito más que irreverente y así arrastrar conmigo a una hilera interminable de estantes para que las mercaderías sean, finalmente, las que me lapiden“ (Eltit 2005 [2002], 52).

Die Darstellung neoliberaler Anomie knüpft Eltit dabei vor allem an die (an)ästhetisierende Erfahrung des Supermarkts durch die Figuren als Ort spezifisch destruktiver Sinneswahrnehmungen. Die fortwährende Ausgesetztheit gegenüber dem Lärm der Kunden, den toxischen Gerüchen und dem grellen Licht sowie der labyrinthischen Struktur des Marktes selbst rufen in den Angestellten nicht nur das Gefühl eines „mareo persistente“ (Eltit 2005 [2002], 50) 
und den schrittweisen Verlust ihrer Wahrnehmungsfähigkeit hervor, ${ }^{20}$ sondern auch eine (Zer-)Störung ihrer menschlichen Subjektivität. Mehrfach beschreiben die Angestellten ihre Selbstwahrnehmung als eine zum Animalischen bzw., genauer, zum Amphibischen tendierende, welche die sukzessive Auflösung einer humanen Welt- und Persönlichkeitsverortung markiert:

Ahora mismo, en medio de una escena torpe y agresiva, me encuentro muy cerca de las
mercaderías, encuclillado. Permanzeco agazapado como si actuara la reencarnación de
un sapo y su ostensible respiración (su miedo) y así, tal como un ente entregado a una
dimensión anfibia, me contengo para no dar un brinco y huir penosamente saltando entre
las piedras en dirección impostergable al agua.
(Eltit 2005 [2002], 49)

Diese Dynamik physischer wie psychischer Degradation, welche Folge einer fortwährend in bellizistischen Metaphern beschriebenen (Kriegs-)Wirtschaft ist, in der die Angestellten gleichermaßen den feindlichen Aufsehern und den Kunden ausgeliefert sind (,este cliente me mira tal como si yo fuese el representante de una casta enemiga que se le ocurrió combatir“, heißt es etwa an einer Stelle (Eltit 2005 [2002], 26)), wird im Roman weiterhin vor allem hinsichtlich ihrer destruktiven Auswirkungen auf zwei fundamentale und eng zusammenhängende anthropologische Dimensionen erfahrbar gemacht: die Wahrnehmung von Zeit und den Gebrauch der Sprache. Der Arbeitstakt der ständigen Mobilisierung führt zur völligen Desorientierung der Figuren, „el tiempo juega de manera perversa conmigo porque no termina de inscribirse en ninguna parte de mi ser. Sólo está depositado en el súper, ocurre en el súper. Se trata de un horario tembloroso e infinito“ (Eltit 2005 [2002], 31). Die Gegenwart nimmt dabei Züge eines unendlichen, durch die zyklischen und monotonen Arbeitsabläufe geprägten Präsens an, während die Fähigkeit zur Erinnerung, zur Memoria, lediglich noch in Form zweckgebundener Mnemotechniken, wie etwa der Anleitung zur Aufstapelung von Waren, nicht mehr aber bezüglich biographischer oder gar kollektiver Erfahrungen existiert. Diese nachhaltige Löschung der Erinnerungsfähigkeit der Figuren, welche an die zentrale politische Rolle des Schweigens im postdiktatorialen Kontexts Chiles gemahnt, wird im Roman mittels eines spezifischen formalen Verfahrens illustriert. Die Rede ist von den zahlreichen Paratexten in Form von Zwischenüberschriften, die mehrheitlich aus Titeln von anarchistischen und sozialistischen Arbeiterzeitungen in Chile zwischen 1900 und 1920 bzw. der Allende-Zeit bestehen und als solche eine allegorische Funktion erfüllen: Sie sind einerseits lesbar als Inschriften, Spuren bzw. Ruinen einer untergegangenen Welt der Arbeiterklasse der industriellen

20 Die Erzählinstanz des ersten Teils formuliert diese anästhesierenden Effekte der Topographie des Supermarkts explizit, wenn es heißt: „estoy a punto de caer anestesiado (con una languidez fatal) en la geografía agujereada del súper“ (Eltit 2005 [2002], 49). 
Moderne, die, wie die sprechenden Titel wie Autonomía y Solidaridad oder Nueva Era anzeigen, nicht nur über eine Kollektividentität und einen geschichtsphilosophischen Optimismus verfügten, sondern vor allem über eine spezifische geteilte Form des Sprechens über die Welt, welche in der neoliberalen Spätmoderne nicht mehr existiert. ${ }^{21}$ Zugleich verweisen sie in ihren historischen Bezugspunkten auf die zwei zentralen Tragödien der chilenischen Arbeitergeschichte im 20. Jahrhundert, nämlich die sog. Matanza de la Escuela Santa María de Iquique, ein Massaker an Salpeter-Arbeitern im Norden Chiles im Jahr 1907, bei dem über 3000 Menschen getötet wurden, sowie den Militärputsch von 1973 - beides Ereignisse, die insbesondere im erinnerungspolitisch auf einem „Pakt des Schweigens“ basierenden postdiktatorialen Chile aus dem kollektiven Gedächtnis verdrängt werden sollten.

Die allegorische Dimension der Paratexte, die als ästhetisches Verfahren in den postdiktatorialen Literaturen des Cono Sur eine zentrale Rolle einnahm (vgl. Avelar 1999), markiert hier also spurhaft jene historische Dimension der Arbeiterklasse als (para-)sozialer Gruppe, deren Distanz zum Prekariat des Neoliberalismus insbesondere im Sprachverlust der Erzählerfiguren der Gegenwart augenfällig wird: „No cuento para nadie“ (Eltit 2005 [2002], 14), bemerkt der anonyme Erzähler gleich zu Beginn des Romans, wobei die Doppeldeutigkeit des Verbs „contar“ (zählen/erzählen) hier die Unmöglichkeit eines neuen kollektiven Narrativs schon anzeigt. Die Sprache bzw. die (aufgrund der synonymen Bedeutung im Spanischen) Zunge werden zu animalischen Fremd-Körpern, die es für das disziplinierte Individuum zu kontrollieren gilt: „no sé qué hacer con la consistencia de mi lengua que crece, se enrosca y me ahoga como un anfibio desesperado ante una injusta reclusión. Me muerdo la lengua. La controlo, la castigo hasta el límite de la herida. Muerdo el dolor" (Eltit 2005 [2002], 16). Darüber hinaus führt die durch die neoliberale Arbeitswelt beförderte Verdinglichung des Menschen nicht nur zu einer Selbstwahrnehmung als maschinenhaftes Objekt ${ }^{22}$ und gezüchtigtes Körper-Ding ${ }^{23}$, sondern vor allem zum genannten Verlust eines Ausdrucksmediums, das zur Benennung der eigenen Ausbeutungsverhältnisse ermächtigen würde. „No me interesa indagar en estos asuntos. No me considero especialmente proclive a pensar en cuestiones abstractas que no conducen a un resultado mensurable“ (Eltit 2005 [2002], 35), bekennt der namenlose Erzähler im ersten Teil und verweist damit implizit auf

21 Für eine ausführliche Analyse dieses Aspekts vgl. auch die Artikel von Draper 2010 und Niebylski 2018.

22 Vgl. Aussagen wie „me radico como un objeto neutro en el pasillo“ (37) bzw. „¿Quién soy?, me pregunto de manera necia. Y me respondo: ,una correcta y necesaria pieza de servicio““ (73).

$23 \mathrm{Vgl}$. „un cuerpo adiestrado para cada una de las tareas de servicio“ (55). 
die sprachliche und ideologische Leere sowie die Unmöglichkeit der Benennung der Klassenverhältnisse im Neoliberalismus.

Infolgedessen bildet der Erzählerdiskurs in Mano de obra in Ermangelung einer kollektiv vermittelbaren Sprache insgesamt drei zentrale sprachliche Register im Rahmen einer (An)Ästhetik des Konsums aus: erstens einen affektiv ,kalten` Duktus, der vor allem über einen distanzierten Erzählerbericht funktioniert, welcher bestimmte affektive oder emotionale Episoden filtert; ${ }^{24}$ zweitens eine Häufung barocker, manierierter Passagen, in denen sich archaische und gelehrte Termini in endlosen Hypotaxengeflechten überlappen und aneinanderreihen und damit bewusst jede mimetische Vorstellung einer zeitgenössischen ,Arbeitersprache‘ verunmöglichen (ein Punkt, auf den am Ende des Artikels noch einmal zurückzukommen ist); und drittens, vor allem im zweiten Teil des Romans in den Gesprächen zwischen den Angestellten des Supermarktes, eine Kommunikation, welche sich außerhalb des Kontrollsystems ihres Arbeitsplatzes nurmehr in Affekten des Aggressiven bzw. des Zorns zu vollziehen vermag.

Dem Roman eignet damit in Analogie zur beschriebenen Unmöglichkeit einer geschichtsphilosophischen Utopie auch hinsichtlich seiner eigenen Form, d. $h$. hinsichtlich fehlender Plotstrukturen oder einer teleologischen Handlung, eine weitreichende Absenz von Narrativität in Form sinn- und orientierungsstiftender Erzählung; vielmehr liegt der Fokus auf einer spezifischen sprachlichen und sensuellen Erfahrbarmachung der (an)ästhetischen Dimensionen der spätmodernen Warengesellschaft mit ihren anomischen Tendenzen. Die Figuren, anhand derer dies geschieht, sind konsequenterweise auch keine komplex angelegten Romanfiguren mit einer eigenen narrativen Identität, sondern ganz im Sinne des etwa von Guy Standing für das Prekariat als typisch postulierten „lack of social identity“ kaum mehr als anonymisierte Repräsentanten eines ausbeuterischen Systems, dessen prinzipielles Merkmal die radikale Verfügbarkeit des prekären Menschen ist. Der súper fungiert damit als paradigmatischer Ort der absoluten Souveränität des Marktes. ${ }^{25}$ Die Figuren - und insbesondere ihre Körper - folgen dem Prinzip des prekären Lebens bzw. des homo sacer im Sinne Agambens als jenem stets radikal - und das heißt bis zur straflosen Versehrung und Tötung - disponiblen

24 Vgl. etwa das wiederholt auftauchende Verfahren, statt direkter bzw. wörtlicher Rede, welche die Leserin in ihrer Expressivität zu affizieren vermöchte, diese Elemente - etwa den Ausdruck von Schmerzempfindungen durch die Figuren - einfach auf abstrakte Weise zu umschreiben und alle Marker einer exzessiv-expressiven Rede zu filtern: „Y luego, con un énfasis teatral, ,los viejos del súper', van señalando, sin un átomo de pudor, sin cesar, sin consideración, la aguda experiencia verbal de cómo transcurren sus dolores“ (Eltit 2005 [2002], 40).

25 Diese absolute Souveränität, die einmal mehr auch auf die Geburt des chilenischen Neoliberalismus aus dem Geist der Militärdiktatur verweist, wird entsprechend in der Schmittschen 
Lebens, das auch in anderen Romanen Eltits wie Fuerzas especiales oder Impuesto a la carne eine zentrale Rolle spielt.

Über diese Beschreibungen des prekären Lebens im chilenischen Neoliberalismus hinaus jedoch thematisieren Eltits Romane immer auch die Frage nach den Möglichkeiten neuer Gemeinschaftsbildungen. Diesbezüglich lässt sich eine durchaus interessante Dynamik innerhalb ihres Werkes ablesen: So scheitert etwa noch in Mano de Obra jegliche Illusion eines solidarischen Arbeiterkollektivs an der letztlich darwinistischen Logik des Überlebenskampfs, welche in der Wohngemeinschaft der Supermarkt-Angestellten herrscht. ${ }^{26}$ Immer wieder wird diese Logik in Bildern des Grotesken ausgeführt, etwa wenn ein alkoholkrankes Mitglied der Gemeinschaft von Enrique, dem Anführer, zum Auflecken seines eigenen Erbrochenen gezwungen wird oder einem drogensüchtigen Mitbewohner das Blut aus der vom Kokain zerstörten Nase auf den Kopf eines Neugeborenen im gleichen Haus tropft. Dieses Klima des Misstrauens führt schließlich nach dem Verrat des Anführers der Gemeinschaft an seinen Kollegen nicht nur zu deren Entlassung, sondern auch zur vollkommenen Zerstörung des fragilen Kollektivs bzw. zur Ent-Hausung aller Bewohner: „Enrique (ahora convertido, después de un ascenso inédito, en el nuevo supervisor de turno) [...] nos borró de las nóminas y nos empujó hasta una extinción dolorosamente dilatada“ (Eltit 2005 [2002], 175).

Einen Ausweg aus der Diktatur des Marktes gibt es in Mano de obra folglich nicht. Statt solidarischer Gesten oder Imaginarien eines alternativen Lebens regiert - ganz im Sinne von Walter Benjamins Thesen vom Kapitalismus als der Zelebrierung eines „Kultus sans rêve et sans merci“‘27 $(1985,100)$ - die Logik der universellen Verschuldung, welche alle Beteiligten stets zur individuellen, nie aber zur systemischen Schuldzuschreibung zwingt - oder wie es lapidar über ein Mitglied der Gruppe heißt, welches sich aus Unachtsamkeit beim Zerteilen von

Formel von der Entscheidungsgewalt über den Ausnahmezustand zitiert, wenn es heißt: „Los supervisores decretaron un turno de emergencia. Sin tregua alguna. Atenderemos [...] a lo largo de 24 horas“ (Eltit 2005 [2002], 69).

26 Es ist allerdings interessant, die werkinterne Dynamik dieses Aspektes bei Eltit zu beobachten, wenn zum Beispiel in ihrem 2013 publizierten Roman Fuerzas especiales über die Polizeigewalt in einem marginalisierten Stadtviertel von Santiago de Chile unter den gleichfalls prekär zusammenlebenden Figuren des Romans durchaus vielfältige Dimensionen solidarischen Handelns bzw. ästhetischer und politischer Auflehnung existieren. Vgl. ausführlich zu diesem Aspekt des Gemeinschaftlichen auch die rezente Studie von Barrientos 2019.

27 Der gesamte Roman ist von dieser Dimension der Ware als Fetisch und des Kapitalismus als Religion durchzogen, etwa wenn es über die Kunden des Supermarktes heißt: „Tocan los productos igual que si rozaran a Dios“ (Eltit 2005 [2002], 5) und der Supermarkt entsprechend als Tempel erfasst wird (Eltit 2005 [2002], 15-16). 
Hühnerschenkeln einen Finger abschneidet: „es culpa de la culiada“ (Eltit 2005 [2002], 155). Die Botschaft des Romans steht damit exemplarisch für einen geschichtsphilosophischen und gleichsam anthropologischen Pessimismus, dessen (An)Ästhetik des Warenkonsums auf vielfältige Weise an Paradigmen der marxistischen Tradition um Benjamin oder Lukács anschließt, ohne dass sich daraus noch wie bei den Theoretikern der Moderne eine wie auch immer geschichtsphilosophisch oder messianisch grundierte Hoffnung auf eine andere Welt jenseits der kapitalistischen Spätmoderne zu eröffnen vermag.

\section{Der Blick der Flâneuse: Der hypermarché als Ort spätmoderner Vergesellschaftung in Annie Ernaux' Regarde les lumières, mon amour (2014)}

Einen ästhetisch wie ideologisch differenten Blick auf die (An)Ästhetiken des Konsums in der spätmodernen Warenwelt liefert die französische Autorin Annie Ernaux in ihrem 2014 erschienenen Buch Regarde les lumières, mon amour. Der Text, der wie alle Bücher der Autorin zwischen soziologischem Essay und autobiographischer Fiktion oszilliert und Teil des von Pierre Rosanvallon initiierten Projekts Raconter la vie ${ }^{28}$ war, ist das Ergebnis einer zwischen November 2012 und Oktober 2013 unternommenen Dokumentation der Besuche der Autorin im hypermarché Auchan ihres Wohnortes in Cergy im Nordwesten von Paris, welcher wiederum Teil des größten Einkaufszentrums der Region ist. Dabei thematisiert Ernaux gleich zu Beginn des Textes den auch hier eingangs erwähnten Aspekt der literarischen Unsichtbarkeit des Supermarkts, der im weiteren Sinne auf die Frage nach der ästhetischen Dignität des Ökonomischen an sich abzielt. Diesbezüglich hat Dominique Viart eine fast generelle Absenz von Sujets der Arbeitswelt in der französischen Literatur zwischen dem Ende des Zweiten Weltkriegs und den 1980er Jahren konstatiert, die insbesondere in den dominanten formexperimentellen und unpolitischen Strömungen wie dem Nouveau Roman begründet gewesen seien (Viart 2013, 139). Ernaux jedoch verweist vor allem auch auf den Zusammenhang zwischen der sozialen Herkunft der Autorinnen der Zeit und den Folgen für die (Ir-)Relevanz bestimmter Sujets und Lebenswelten,

28 Vgl. dazu ausführlich auch den Artikel von Robert Lukenda 2017, dem an dieser Stelle dafür gedankt sei, mich überhaupt erst auf den Text von Ernaux aufmerksam gemacht zu haben. 
denn: „jusqu'aux années 1970, les écrivains [...] étaient majoritairement d'origine bourgeoise et vivaient à Paris où les grandes surfaces n'étaient pas implantées. (Je ne vois pas Alain Robbe-Grillet, Nathalie Sarraute ou Françoise Sagan faisant des courses dans un supermarché, Georges Perec si, mais je me trompe peut-être)“ (2014, 43). Neben der Dimension der Klasse führt Ernaux außerdem die Kategorie des Geschlechts an, die gleichfalls zur literarischen Marginalisierung des Ökonomischen bzw. der Warenwelt beigetragen habe, denn „ce qui relève du champ d'activité plus ou moins spécifique des femmes est traditionnellement invisible, non pris en compte, comme d'ailleurs le travail domestique qu'elles effectuent. Ce qui n'a pas de valeur dans la vie n'en a pas pour la littérature“ (2014, 43). Vor diesem Hintergrund wird Ernaux' Supermarkt-Projekt zu einer von Beginn klassen- und gender- und damit auch stilkritischen Unternehmung, die gerade in ihrer Problematisierung herrschender ästhetischer Normen deren anästhetisierende Macht im Sinne einer Ausblendung bestimmter Themenbereiche und Schreibweisen erkennbar macht. Der hypermarché wird aus dieser Perspektive zu einem privilegierten Beobachtungsort spätmoderner Vergesellschaftungsprozesse, ist er doch - mit Ausnahme der „habitants du centre de Paris et des grandes villes anciennes“ - für die restliche Bevölkerung Frankreichs „un espace familier dont la pratique est incorporée à l'existence, mais dont on ne mesure pas l'importance sur notre relation aux autres, notre façon de ,faire société، avec nos contemporains au XXI ${ }^{\mathrm{e}}$ siècle“ (Ernaux 2014, 12).

Die spezifische, an der Soziologie Bourdieus ${ }^{29}$ geschulte Ästhetik von Ernaux’ „ethnotextes“ (Oliver 2011, 180) setzt daher - im Unterschied zur barocken Überwältigungsästhetik bei Diamela Eltit - konsequent auf die explizite Thematisierung der Bedingungen der Wahrnehmung des Sozialen. „Je ne vois des gens que leur corps, leur apparence, leurs gestes. Ce qu'ils mettent dans leur panier, leur caddie. J'en déduis plus ou moins leur niveau de vie“ (Ernaux 2014, 32), heißt es in der an Bourdieus grundlegende Hypothese angelehnten Perspektive auf die Struktur des Sozialen in La distinction, welche bekanntlich „par la structure des relations entre toutes les propriétés pertinentes qui confère à chacune d'elles et aux effets qu'elle exerce sur les pratiques, leur valeur propre“ (Bourdieu 1979, 118)

29 Vgl. Zur Bedeutung von Bourdieus Denken für Ernaux’ Ästhetik auch die Bemerkungen der Autorin über den „nouvel apprentissage du regard et de l'oreille“, den die Lektüre Bourdieus für sie bedeutet habe und der fundamental für die Entwicklung einer eigenen Schreibweise gewesen sei: „II m’a aidée à concevoir ce que j’appelle ,l'écriture distanciée‘ (plutôt que ,plate'), depuis ma place d'écartelée entre deux mondes sociaux. Il m’a renforcée dans ma détermination à chercher une voie d'écriture entre le personnel et l'impersonnel, qui prenne en compte la ,rumeur““ (Ernaux 2013, 26-27). Vgl. zur soziologischen Dimension bei Ernaux auch den Artikel von Meizoz 2012. 
bestimmt ist. Zugleich aber sei ihr Text, betont Ernaux, „[p]as d'enquête ni d'exploration systématiques“ über die Warenwelt des hypermarché, sondern „un journal [...]. Un relevé libre d'observations, de sensations, pour tenter de saisir quelque chose de la vie qui se déroule là“ $(2014,15) .{ }^{30}$ Die Perspektive Ernaux’ ist folglich explizit die der Flâneuse, die sich in die Menschen- und Warenflüsse wirft, welche sich von den modernen Stadt- in die spätmodernen Einkaufszentren verlagert haben und der Autorin zugleich einen Ort der Distraktion bieten: „Je me suis souvent jetée au centre commercial pour oublier l'insatisfaction de l'écriture en me mêlant à la foule des acheteurs et des flâneurs [...] tous ceux qui y viennent comme autrefois, en ville - faire un tour“ (2014, 44). Das Einkaufszentrum und der Supermarkt werden damit - wiederum in Unterschied zur Darstellung bei Eltit - vor allem als Ort wahrgenommen, an dem Menschen unterschiedlicher sozialer Hintergründe aufeinandertreffen und an dem die Beobachterin selbst essentieller Teil dieses Sozialen ist, wird sie doch ebenfalls von allen anderen beobachtet (vgl. Ernaux 2014, 40). Der Supermarkt ist für Ernaux eine paradigmatische mise en abyme der Gesellschaft, die lediglich denen verschlossen bleibt, die aus habituellen Gründen diese Orte nie aufsuchen: „Les femmes et les hommes politiques, les journalistes, les ,experts', tous ceux qui n'ont jamais mis les pieds dans un hypermarché ne connaissent pas la réalité sociale de la France d'aujourd'hui“" $(2014,12) .^{31}$

Der Supermarkt wird aus dieser Perspektive zu einem ambivalenten Ort, an dem sich sowohl die sozialen Ungerechtigkeiten besichtigen als auch ein Gefühl der gesellschaftlichen und menschlichen Verbundenheit erleben lassen, wie Ernaux festhält: „Souvent, j’ai été accablée par un sentiment d'impuissance et d'injustice en sortant de l'hypermarché. Pour autant, je n'ai cessé de ressentir l'attractivité de ce lieu et de la vie collective, subtile, spécifique, qui s’y déroule“ (2014, 71). Aus diesem Grund versteht Ernaux den Supermarkt auch nicht vorwiegend negativ als reinen Tempel des Konsums wie in der Tradi-

30 Diese Reflexion über Wahrnehmungsfragen schließt auch die exakte Benennung aller relevanten Marker sozialer Existenz mit ein, wie etwa deutlich wird, wenn Ernaux an einer Stelle darüber sinniert, ob sie eine von ihr beobachtete Kundin als „femme noire“ bezeichnen oder diese Angabe aus vermeintlich politisch korrekten Gründen unterlassen solle, wogegen sich Ernaux ausspricht, denn: „C'est en somme ,blanchir“ implicitement cette femme puisque le lecteur blanc imaginera, par habitude, une femme blanche. C'est refuser quelque chose de son être et non des moindres, sa peau. Lui refuser textuellement la visibilité. Exactement l'inverse de ce que je veux faire, de ce qui est mon engagement d'écriture: donner ici aux gens, dans ce journal, la même présence et la même place qu'ils occupent dans la vie de l'hypermarchéc“ (2014, 22).

31 Vgl. zu dieser elitenkritischen Position Ernaux' auch ihre wiederholt positiven Aussagen zur Sozialbewegung der Gilets jaunes. 
tion der marxistischen Warenkritik à la Adorno oder als einen sog. ,non-lieu' im Sinne Marc Augés; vielmehr geht es ihr um eine Gegenerzählung zu diesen soziologischen Positionen, die letztlich mehr oder weniger unterschwellig die bourgeoisen Vorbehalte gegen die Lebenswelt der ,einfachen Leute' auf einer soziologisch sublimierten Ebene zu teilen scheinen. In diesem Sinne formuliert Ernaux das Ziel ihres Buchs als ein ,rendre compte d'une pratique réelle de leur fréquentation, loin des discours convenus et souvent teintés d'aversion que ces prétendus non-lieux suscitent et qui ne correspondent en rien à l'expérience que j'en ai“ (2014, 12-13). Der Supermarkt ist für Ernaux kein ,non-lieu‘, sondern vielmehr ein ,lieu de mémoire‘, ein Erinnerungsort von biographischen Szenerien des Alltäglichen, welche wiederum Teil einer geteilten kollektiven Erfahrung sind, an deren Prägung gerade die Kunst mitzuwirken habe, denn „quand je regarde derrière moi, je me rends compte qu'à chaque période de ma vie sont associées des images de grandes surfaces commerciales, avec des scènes, des rencontres, des gens“ (2014, 10). Folgerichtig ist Ernaux’ Gegenwartsbeobachtung des Supermarkts durchzogen von autobiographischen Fragmenten, die von ihrer ersten eigenen Supermarkterfahrung als Au-pair-Mädchen im London der 1960er über die Hamsterkäufe während der Proteste von 1968 bis zu einem Besuch im ersten slowakischen Supermarkt der post-kommunistischen Zeit Anfang der 1990er Jahre und der Beobachtung der beginnenden éducation consommatrice der dortigen Bevölkerung reichen. Aus dieser Perspektive sind der Supermarkt und seine Warenwelt eben kein reiner Funktionsort oder die Bühne zur Produktion des falschen Scheins der Konsumsphäre, sondern ein von ,echten' Affekten und Erinnerungen durchzogener Raum, in dem gerade innerhalb der vermeintlich opaken Welt der Dinge Formen emotionaler Vergemeinschaftungen und Begegnungen vollzogen werden:

Les super et hypermarchés ne sont pas réductibles à leur usage d'économie domestique, à la ,corvée des courses'. Ils suscitent des pensées, fixent en souvenirs des sensations et des émotions. On pourrait certainement écrire des récits de vie au travers des grandes surfaces commerciales fréquentées.

(Ernaux 2014, 11)

Trotz dieser Aufwertung des Supermarkts ist Ernaux' journal auch eine kritische Reflexion über die problematischen Dimensionen der Konsumwelt und die darin wirksamen Dimensionen von Ästhetik und Anästhetik. Immer wieder rekurriert der Text auf die Funktionsweisen des ästhetischen Kapitalismus zur Erweckung und Steuerung der Begierden, die vom architektonischen Design des Einkaufszentrums mit seinen „portiques dont quelques-uns, monumentaux, évoquent l'entrée d'un temple mi-grec mi-asiatique“ (Ernaux 2014, 14) bis zum beständig wechselnden Warenangebot in seiner entsprechenden Darbietung reichen, ganz im Sinne jenes Prinzips der „grande distribution qui fait la loi dans nos envies“ (Ernaux 
2014, 41). Wiederholt verweist Ernaux dabei gerade auf die anästhetischen Dimensionen der Waren- und Konsumästhetik, indem sie die Funktionsweisen des Wahrnehmens im Supermarkt als solche explizit macht, etwa wenn es über die Bewerbungsformen im Weihnachtsgeschäft heißt: „Les prix sont en lettres gigantesques, toujours sur le même fond jaune acide. Je m’aperçois que cette démesure agit de façon hypnotique, je serais prête à croire que ces poissons sont littéralement donnés“ (2014, 34).

Zugleich wird der Supermarkt in seinen Kontrolldimensionen und seiner Topographie sowie der daraus resultierenden spezifischen Zeitwahrnehmung auf ganz ähnliche Weise beschrieben wie bei Eltit: Das Einkaufszentrum ist eine gleichsam gegen den Rest der Stadt abgeschlossene, künstliche Eigenwelt, eine Art ,paragesellschaftliche“ Sphäre, „une énorme forteresse rectangulaire en briques rouge brun“ (Ernaux 2014, 13) mit einer labyrinthischen Struktur, die einerseits nach den Gesetzen der Dynamisierung des Kapitalismus im Sinne eines ständigen Wechsels der Angebote funktioniert, andererseits aber gerade in diesen sich ewig wiederholenden Konsumschleifen auf die Aufhebung jeglichen Zeitempfindens abzielt: „Dans le centre commercial, on ne voit pas le temps. Il n'est pas inscrit dans l'espace. Il ne se lit nulle part. Il y a replacement des boutiques, rotations des rayons, renouvellement des marchandises, du nouveau qui ne change fondamentalement rien“ (Ernaux 2014, 33). Der daraus resultierende Effekt entspricht dabei der Diagnose Eltits: Die Zeitlogik der kapitalistischen Konsumsphäre zielt - als ihr vielleicht wichtigster anästhetischer Effekt - auf die Aufhebung der Möglichkeit von Geschichte selbst. „Qu'il n'y a pas d'Histoire“ (Ernaux 2014, 42), notiert Ernaux diesen Eindruck, der wie bei Eltit zugleich das Ende aller geschichtsphilosophischen Utopien und Veränderung jenseits der ewigen kapitalistischen Gegenwart markiert. Das Einkaufszentrum fungiert auch bei Ernaux als Verkörperung der absoluten Souveränität des Marktes, die über ein für den spätmodernen Kapitalismus typisches System einer „liberté constamment surveillé“ $(2014,67)$ herrscht. Innerhalb seiner Grenzen ist das Einkaufszentrum folglich nicht nur ein Ort totaler und von den Kunden bereits internalisierter Kontrolle - „propriété d'un groupe privé, il est entièrement fermé, surveillé et nul ne peut y pénétrer en dehors d'horaires déterminés“ (Ernaux 2014, 14) -, sondern zugleich ein Ort mit gesellschaftlicher Ordnungsmacht. Diese funktioniert explizit durch die Verdrängung all jener, die in der Logik des Konsums keinen Wert besitzen, namentlich der Obdachlosen, denen der Zutritt zur Warenwelt bewusst verwehrt wird: „Il y a de plus en plus de SDF dans l'ensemble de la société mais de moins en moins autour du centre commercial, à l'exception de deux endroits, qui ne font pas partie du territoire privé du centre commercial“ (Ernaux 2014, 39). Sie funktioniert aber zugleich auch auf eine implizite Weise, indem über die Distinktionsmacht 
der Waren selbst - und hier wird Ernaux' ,bourdieuscher Blick` am deutlichsten die gesellschaftlichen (Klassen-)Unterschiede markiert werden. Die Unterteilung der Sektionen des Supermarkts in Billig- und Hochpreissegmente und der Blick aufs Kassenband machen den Ort des Konsums zum Schaufenster der feinen (und groben) Unterschiede der Gesellschaft und unterstreichen die zugleich vergemeinschaftende und separierende Macht der Dinge:

[J]'ai mesuré de plus en plus la force du contrôle que la grande distribution exerce dans ses espaces de façon réelle et imaginaire - en suscitant les désirs aux moments qu'elles détermine -, sa violence, recelée aussi bien dans la profusion colorée des yaourts que dans les rayons gris du super-discount. Son rôle dans l'accommodation des individus à la faiblesse des revenus, dans le maintien de la résignation sociale.

(Ernaux 2014, 71)

Die Darstellung des Supermarkts und der Warenwelt ist bei Ernaux, so lässt sich zusammenfassen, von einer grundlegenden Ambivalenz geprägt: Einerseits wird immer wieder positiv die vergemeinschaftende Funktion des Marktes herausgestellt, „[l]e journal municipal m'apprend que 130 nationalités sont présentes sur l'ensemble du territoire de Cergy. Nulle part ailleurs elles ne se côtoient autant qu'au centre commercial des Trois-Fontaines, à Auchan“ (2014, 38); andererseits werden die problematischen Dimensionen der gegenwärtigen Konsumwelt ausführlich thematisiert, die über die genannte Klassendimension hinaus auch etwa Aspekte globaler Ausbeutung in den Textilfabriken von Bangladesch oder die Reproduktionsmacht des Marktes hinsichtlich bestimmter Geschlechterklischees umfassen. Eindeutig hingegen ist die pessimistische Position Ernaux' gegenüber den Möglichkeiten einer anderen Gesellschaft jenseits der spätmodernen Marktgesellschaft. „M'est venue la question que je me pose des quantités de fois, la seule qui vaille: pourquoi on ne se révolte pas?“ (Ernaux 2014, 67), fragt Ernaux nach den Möglichkeiten des Aufstands gegen das System. Die Antwort ist eindeutig und einmal mehr in der ästhetischen (Über-)Macht des Marktes zur unendlichen Produktion von „Begehrnissen“ begründet, die in letzter Instanz alle Energiereservoirs einer potentiellen Revolte zu anästhesieren vermag: „Tous trop fatigués, et bientôt nous serions dehors, enfin sortis de la nasse, oublieux, presque heureux. Nous sommes une communauté de désir, non d’action“ (Ernaux 2014, 67). 


\section{$5 \mathrm{Wa}(\mathrm{h})$ re Repräsentationen? Aporien einer (An)Ästhetik des Konsums der Spätmoderne}

Die für die Spätmoderne soziologisch vielfach postulierte „Krise des Allgemeinen auf den Ebenen der sozialen Anerkennung, der Allgemeinheit der Kultur und des Politischen“ (Reckwitz 2017b, 437) hat bekanntlich nicht nur tiefgreifende Folgen für die Formen und Regeln politischer, sondern auch künstlerischer Repräsentation. Wenn die Gesellschaft als solche nur noch in Form sich beständig differenzierender Teilgruppen funktioniert und sie damit zunehmend $\mathrm{zu}$ einer, wie Pierre Rosanvallon schreibt, „société illisible“ $(2014,17)$ wird - welche Möglichkeiten und Formen ästhetischer Darstellung stehen dann etwa der Literatur noch zur Verfügung, um über einen Kollektivsingular wie ,Gesellschaft‘ überhaupt zu sprechen bzw. eine Repräsentationsarbeit leisten zu können, die sich nicht selbst automatisch in den ,Dienst“ bestimmter künstlerischer und/oder (identitäts-) politischer Interessen sozialer Teilgruppen stellen will? Diese Frage führt theoretisch wie historisch zu weit, als dass sie an dieser Stelle angemessen diskutiert werden könnte. Sie zielt jedoch in letzter Instanz auf eine Darstellungsproblematik, welche die Literatur seit jeher begleitet hat und in der Moderne von besonderer Relevanz wurde, nämlich, wie denn gerade „Personen, Ereignisse und alltägliche Existenzbedingungen (,Milieus'), die die längste Zeit unterhalb der Schwelle der Darstellung verharrten oder denen allenfalls ein subalterner Platz [...] zugestanden wurde“ nicht nur soziologisch, sondern eben auch im Medium der Literatur und insbesondere durch „Einsatz ,realistischer“ oder ,naturalistischer“ Künste“ (Balke 2019, 21) symbolisch repräsentierbar gemacht werden konnten.

Die beiden (an)ästhetischen Register, welche die Texte von Diamela Eltit und Annie Ernaux diesbezüglich liefern, könnten unterschiedlicher kaum sein: So schließt Eltit in Mano de obra an eine modernistisch bzw. avantgardistisch grundierte (An)Ästhetik an, ${ }^{32}$ deren formale Grundoperation eine „vertiginous fragmentation [that] shatters of experience no longer representable as coherent wholes or as symbolic totalities“ ist, während die Sprache einer „de-anecdotalization that defies paraphrase“ unterzogen wird (Avelar 1999, 164 bzw. 173). Die Darstellung der spätmodernen Konsum- und Warenwelt am Beispiel des chilenischen Supermarkts unterliegt daher bei Eltit einem ästhetischen wie politischen Paradox: Ästhetisch gesprochen erzeugt der Text in seinem mäandernden, barocken Sprachwulst und den beständig aufeinanderprallenden Registern

32 Eltit begann ihre künstlerische Karriere als Mitglied des chilenischen Neoavantgarde-Kollektivs CADA, welches in den 1980er Jahren vor allem durch Performances und Interventionen im öffentlichen Raum des diktatorischen Chiles für Aufsehen sorgten. 
des Archaischen, des Vulgären und des Sozioanalytischen einen Diskurs, der bewusst jede mimetische Vorstellung einer zeitgenössischen ,Arbeitersprache` oder einer an traditionelle realistische Codes geknüpften littérature engagée konterkariert; zugleich aber eignet dem Text gerade durch die bisweilen anästhesierende Wirkung der Lektüre, die durch die Verwirrung und Desorientierung in der Leserin zustande kommt, indem ihr nämlich zeitliche, räumliche oder figurenbezogene Ankerpunkte konstant verweigert werden, eine Art mimetischer bzw. realistischer Effekt. Diese sinnliche Überforderung durch die bewusste Verweigerung narrativer Ordnung und Sinnhaftigkeit macht eben den für die Arbeit im Supermarkt konstitutiven ,Wahrnehmungsterror' erfahrbar, wodurch die (An)Ästhetik Eltits - ganz im Sinne der historischen avantgardistischen Logik - die Überwältigungs- und Gewaltelemente ihres Beschreibungsgegenstandes in ihre Formen- und Darstellungssprache integriert. ${ }^{33}$ Politisch gelesen ergibt sich daraus die Aporie, dass diese höchst kritische, ja engagierte Darstellung der sozialen Abgründe hinter den bunten Oberflächen des neoliberalen Kapitalismus der Spätmoderne auf eine literarische (An)Ästhetik zurückgreift, die - wie das gesamte Werk Eltits - bestenfalls von einem akademischen (bourgeoisen) Publikum zu verstehen bzw. zu goutieren ist. Wenn also etwa Héctor Hoyos in Eltits Arbeiterdarstellung die Möglichkeit „of seizing that tension ,from below', recreating discrete experiences and putting us in the shoes of others“ $(2015,124)$ sieht, dann stellt sich immer schon die Frage, welche

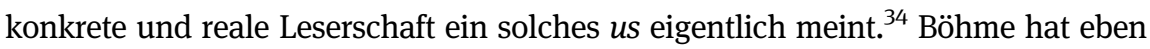

33 Auch Welsch hält (allein?) eine solche modernistische (An)Ästhetik, die eben an benjaminsche Kategorien anschließt, als paradigmatische (Erkenntnis-)Form zur Durchbrechung des schönen Scheins für geeignet: „Eine solche anästhetisch akzentuierte Ästhetik würde zu einer Schule der Andersheit. Blitz, Störung, Sprengung, Fremdheit wären für sie Grundkategorien. Gegen das Kontinuum des Kommunizierbaren und gegen die schöne Konsumption setzte sie auf Divergenz und Heterogenität [...] Werke der Verweigerung [...] deren Aneignung fehlschlägt“ (2017, 43-44). Zu einer kritischen Lektüre dieser avantgardistischen Ästhetiken im chilenischen Kontext vgl. auch den einschlägigen Aufsatz von Thayer 2006.

34 Vgl. dazu auch Eltits Aussage zu ihren Zweifeln über die politischen Wirkmöglichkeiten von Literatur bzw. ihre bewusste Distanzierung vom Markt bzw. den Institutionen des Literaturbetriebs (welche sie andererseits mit zahlreichen internationalen und nationalen Preise und Ehrendoktorwürden überhäuft haben): „No creo sinceramente que la literatura pueda ocasionar una conmoción política. Más bien la posibilidad de una conmoción proviene del impulso del conjunto de la esfera social y en su interior cabe la literatura como un acto más. En mi caso particular, desde mis comienzos, me importó mantener una política de escritura y me he mantenido bastante alejada del mercado o de las modas. Y por otro lado tengo un imaginario al que no puedo ni quiero renunciar que cae y recae sobre ciertos espacios, pero no se trata de una voluntad sino más bien de la invasión una fuerza deseante que me excede. Pero lo que sí pienso de manera férrea es que la literatura tiene el poder de modificar políticamente a la es- 
diese Aporie, der Eltits Werk unterliegt, präzise an Adornos ästhetischer Theorie diskutiert: Die typische modernistische Hoffnung auf eine Versöhnung der gesellschaftlichen Gegensätze in der Kunst und zugleich gegen die Kulturindustrie sei eine „kaum zu erfüllende Forderung. Denn dafür müsste das mimetische Moment so spröde oder so reflexiv gemacht werden, dass es dann wiederum nur noch der Bildungsschicht zugänglich wäre“ (Böhme 2018, 97).

Der Text von Annie Ernaux setzt demgegenüber auf eine andere Strategie: Im Gegensatz zur modernistischen ,Schock-Wirkung'von Eltits Roman basiert ihr journal gerade auf einer absoluten sprachlichen Zugänglichkeit und Transparenz des literarischen Textes, welches über eine räumlich, zeitlich und autobiographisch konkrete sowie radikal intelligible Darstellung der Bedingungen von Wahrnehmungsprozessen eine ästhetische Form entwirft, die auch und gerade von ,einfachen' Leserinnen problemlos rezipiert werden kann. Diese in Ernaux' écriture plate angelegte anti-modernistische Stoßrichtung ist, wie argumentiert wurde, bewusst gegen ein bestimmtes elitäres Literaturverständnis der Moderne und seine thematischen, formalen und sozialen Sanktionsund Exklusionsmechanismen gerichtet. Die Darstellung der Konsumwelt bleibt bei Ernaux folglich von „Widersprüchen nicht unberührt, nicht nur, weil sie selbst durch ihr Kaufverhalten dazu beiträgt, sie unaufgelöst bestehen zu lassen, sondern auch, weil die Kritik an der Logik des Konsums und an dem von dieser getragenen Dispositiv der Macht nicht verhindert, dass der Gedanke an den Supermarkt für sie positiv besetzt ist“ (Urban 2018, 156). In dieser Ausstellung der Widersprüchlichkeit menschlicher Existenz in der spätmodernen Warenwelt jedoch vermag Ernaux' Darstellung womöglich einen ,realistisch(er)en' Effekt dadurch zu erzielen, dass sie die unbestreitbare vergemeinschaftende Macht der Waren und des Konsums nicht leugnet, sondern stattdessen die ambivalenten Effekte dieser Tatsache zum Gegenstand ihres Schreibens macht. ${ }^{35}$ Eltits Roman dagegen schließt sowohl ideologisch als auch ästhetisch an Elemente der klassischen marxistischen Waren- und Kunstkritik an, in denen der Supermarkt ausschließlich als Bühne der kapitalistischen Ausbeutung und die modernistisch-avantgardistisch geschulte Kunst als (vermeintlich) widerständiger Hort des Nicht-Konsumierbaren gegen die Kulturindustrie zu fungieren haben. Die Frage, welche dieser (An)Ästhetiken letztlich die geeignetere Form zur $\mathrm{Wa}(\mathrm{h})$ ren-Repräsentation im neoliberalen Kapitalismus ,unserer‘ Spätmoderne ist, dürfte letzteren gleichwohl unberührt

fera literaria misma: lo hace ensanchando los límites impuestos y problematizando las convenciones. Esa es su gran misión política“ (Locane/Loy 2015, 69).

35 Vgl. dazu auch die treffende Bemerkung von Drügh: „Es ist in unseren Überflussgesellschaften nur um den Preis der Unaufrichtigkeit möglich, in third person-Manier über unsere Identität als Konsumenten zu sprechen“ $(2015,19)$. 
lassen, solange beide Bücher in angemessener Lieferzeit über die entsprechenden Plattformen und gegen Zahlung des Produktpreises bezogen und konsumiert werden können.

\section{Bibliographie}

Alwort, David J. „Supermarket Sociology“. New Literary History 41.2 (2010): 301-327.

Avelar, Idelber. The Untimely Present: Postdictatorial Latin American Fiction and the Task of Mourning. Durham, NC: Duke University Press, 1999.

Balke, Friedrich. „Den Tumult dokumentieren. Zum politischen Einsatz der Mimesis bei Auerbach“. Im Gedränge. Figuren der Menge. Hg. Hermann Doetsch und Cornelia Wild. Paderborn: Fink, 2019. 19-39.

Barrientos, Mónica. La pulsión comunitaria en la obra de Diamela Eltit. Pittsburgh, PA: Latin American Research Commons, 2019.

Baudrillard, Jean. La société de consommation. Paris: Gallimard, 2017 [1970].

Beckert, Jens. Imagined Futures. Fictional Expectations and Capitalist Dynamics. Cambridge, MA: Harvard University Press, 2016.

Benjamin, Walter. Das Passagen-Werk. Frankfurt a.M.: Suhrkamp, 1983.

Benjamin, Walter. „Kapitalismus als Religion“. Walter Benjamin. Gesammelte Schriften (Bd. 6). Hg. Rolf Tiedemann und Hermann Schweppenhäuser. Frankfurt a.M.: Suhrkamp, 1985. 100-103.

Böhme, Gernot. Ästhetischer Kapitalismus. Berlin: Suhrkamp, 2018.

Boltanski, Luc und Arnaud Esquerre. Bereicherung. Eine Kritik der Ware. Berlin: Suhrkamp, 2019.

Bourdieu, Pierre. La distinction. Critique sociale du jugement. Paris: Les Éditions de Minuit, 1979.

Bowlby, Rachel. Carried Away: The Invention of Modern Shopping. New York, NY: Columbia University Press, 2001.

Chamayou, Grégoire. La société ingouvernable. Une généalogie du libéralisme autoritaire. Paris: La Fabrique éditions, 2018.

Draper, Susanna. „The Question of Awakening in Postdictatorship Times: Reading Walter Benjamin with Diamela Eltit“. Discourse 32.1 (2010): 87-116.

Drügh, Heinz. Ästhetik des Supermarkts. Konstanz: Konstanz University Press, 2015.

Eiden-Offe, Patrick. „Marxismus und Kritische Theorie“. Handbuch Literatur \& Ökonomie. Hg. Joseph Vogl und Burkhardt Wolf. Berlin: De Gruyter, 2020. 3-17.

Eltit, Diamela. Mano de obra. Santiago de Chile: Seix Barral, 2005 [2002].

Eltit, Diamela. Fuerzas especiales. Cáceres: Periférica, 2015 [2013].

Ernaux, Annie. „La preuve par corps“. Bourdieu et la littérature. Hg. Jean-Pierre Martin. Nantes: Cécile Defaut, 2010. 24-27.

Ernaux, Annie. Regarde les lumières, mon amour. Paris: Seuil, 2014.

García Canclini, Néstor. Consumidores y ciudadanos. Conflictos multiculturales de la globalización. Mexiko-Stadt: Grijalbo, 1995.

Hoyos, Héctor. Beyond Bolaño. The Global Latin American Novel. New York: Columbia University Press, 2015. 
Klein, Naomi. The Shock Doctrine: The Rise of Disaster Capitalism. New York: Metropolitan Books, 2007.

Koschorke, Albrecht. Wahrheit und Erfindung. Grundzüge einer Allgemeinen Erzähltheorie. Frankfurt a.M.: Fischer, 2012.

Lessenich, Stephan. Neben uns die Sintflut: Die Externalisierungsgesellschaft und ihr Preis. Berlin: Hanser Berlin, 2016.

Locane, Jorge und Benjamin Loy. „,En Chile se paga hasta por respirar. La palabra gratuidad infunde terror‘. Una conversación con Diamela Eltit“. alba. lateinamerika lesen 8 (2015): 67-71.

Loy, Benjamin. „Fragile Parallelitäten: Neoliberalismus, Poststaatlichkeit und Gewalt im mexikanischen Gegenwartskino“. Parallel- und Alternativgesellschaften in der Gegenwartsliteratur. Hg. Teresa Hiergeist. Würzburg: Könighausen \& Neumann, 2017. 105-139.

Lukács, Georg. Geschichte und Klassenbewusstsein. Studien über marxistische Dialektik. Darmstadt: Luchterhand, 1983 [1923].

Lukenda, Robert. „Raconter la vie, représenter et déchiffrer la société - neue sozialepistemologische ,Allianzen“ und literarische Antworten auf gesellschaftliche Zerfallsprozesse in Frankreich“. Parallel- und Alternativgesellschaften in der Gegenwartsliteratur. Hg. Teresa Hiergeist. Würzburg: Königshausen \& Neumann, 2017. 77-103.

Marchart, Oliver. Die Prekarisierungsgesellschaft. Prekäre Proteste. Politik und Ökonomie im Zeichen der Prekarisierung. Bielefeld: transcript, 2013.

Marx, Karl. Das Kapital. Kritik der politischen Ökonomie (Bd. 1). MEW 23. Berlin: Dietz, 1962 [1867].

Mazzucato, Mariana. The Value of Everything. Making and Taking in the Global Economy. London: Penguin, 2019.

Meizoz, Jérôme. „Annie Ernaux: posture de l'auteure en sociologue.“ Annie Ernaux: se mettre en gage pour dire le monde. Hg. Thomas Hunkeler und Marc-Henry Soulet. Genf: Metis, 2012. 27-44.

Moulian, Tomás. Chile actual. Anatomía de un mito. Santiago de Chile: LOM, 2002.

Neumann, Birgit (Hg.). Präsenz und Evidenz fremder Dinge im Europa des 18. Jahrhunderts. Göttingen: Wallstein, 2015.

Niebylski, Dianna. „Gramáticas capitalistas, retóricas contrahegemónicas y la prensa obrera chilena: Mano de obra de Diamela Eltit“. Pobreza y precariedad en el imaginario latinoamericano del siglo XXI. Hg. Stephen Buttes und Dianna Niebylski. Santiago de Chile: Cuarto propio, 2017. 415-457.

Niebylski, Dianna. „Ironía y violencia en Mano de obra y Fuerzas especiales de Diamela Eltit“. Ironía y violencia en la literatura latinoamericana. Hg. Brigitte Adriaensen und Carlos van Tongeren. Pittsburgh: Instituto Internacional de Literatura Iberoamericana, 2018. 59-94.

Oliver, Annie. „,Instantanés de la vie quotidienne‘: la vie matérielle selon Annie Ernaux“. Écrire le présent. Hg. Gianfranco Rubino und Dominique Viart. Paris: Armand Collin, 2013. 173-184.

Polanyi, Karl. The Great Transformation. The Political and Economic Origins of Our Time. Boston: Beacon Press, 2001 [1944].

Prinz, Sophia. „Einleitung: Kulturelle Ökonomie und neue Formen der Kritik“. Ästhetik und Gesellschaft. Grundlagentexte aus Soziologie und Kulturwissenschaften. Hg. Andreas Reckwitz, Sophia Prinz und Hilmar Schäfer. Berlin: Suhrkamp, 2017. 353-369.

Rancière, Jacques. Politik der Literatur. Wien: Passagen, 2011. 
Reckwitz, Andreas. „Ästhetik und Gesellschaft - ein analytischer Bezugsrahmen“. Ästhetik und Gesellschaft. Grundlagentexte aus Soziologie und Kulturwissenschaften. Hg. Andreas Reckwitz, Sophia Prinz und Hilmar Schäfer. Berlin: Suhrkamp, 2017. 13-54.

Reckwitz, Andreas. Die Gesellschaft der Singularitäten. Zum Strukturwandel der Moderne. Berlin: Suhrkamp, 2017b.

Rosa, Hartmut. Resonanz. Eine Soziologie der Weltbeziehung. Berlin: Suhrkamp, 2019.

Rosanvallon, Pierre. Le Parlement des invisibles. Paris: Seuil, 2014.

Schlünder, Susanne und Andrea Stahl (Hg.). Affektökonomien. Konzepte und Kodierungen im 18. und 19. Jahrhundert. Paderborn: Fink, 2018.

Schönthaler, Philipp. Portrait des Managers als junger Autor. Zum Verhältnis von Wirtschaft und Literatur. Eine Handreichung. Berlin: Matthes \& Seitz Berlin, 2016.

Schrage, Dominik. Die Verfügbarkeit der Dinge. Eine historische Soziologie des Konsums. Frankfurt a.M.: Campus, 2009.

Slobodian, Quinn. Globalists. The End of Empire and the Birth of Neoliberalism. Cambridge, MA: Harvard University Press, 2018.

Standing, Guy. The Precariat. The New Dangerous Class. London: Bloomsbury, 2016.

Viart, Dominique. „Écrire le travail. Vers une sociologisation du roman contemporain?“. Écrire le présent. Hg. Gianfranco Rubino und Dominique Viart. Paris: Armand Collin, 2013. 133-156.

Vogl, Joseph. Das Gespenst des Kapitals. Zürich: Diaphanes, 2010.

Vogl, Joseph, und Burkhardt Wolf (Hg.). Handbuch Literatur \& Ökonomie. Berlin: De Gruyter 2020.

Weber, Max. Rationalisierung und entzauberte Welt. Schriften zu Geschichte und Soziologie. Leipzig: Reclam, 1989.

Welsch, Wolfgang. „Ästhetik und Anästhetik“. Ästhetisches Denken. Hg. Wolfgang Welsch. Stuttgart: Reclam, 2017. 11-45. 
